\title{
Glutathione supplementation suppresses muscle fatigue induced by prolonged exercise via improved aerobic metabolism
}

Wataru Aoi ${ }^{1 *}$, Yumi Ogaya ${ }^{1}$, Maki Takami ${ }^{1}$, Toru Konishi ${ }^{2}$, Yusuke Sauchi $^{2}$, Eun Young Park ${ }^{3}$, Sayori Wada ${ }^{1}$, Kenji Sato ${ }^{4}$ and Akane Higashi ${ }^{1}$

\begin{abstract}
Backgrounds: Glutathione is an endogenous redox couple in animal cells and plays important roles in antioxidant defense and detoxification, although it is unknown if oral glutathione supplementation affects exercise-induced physiological changes. The present study investigated the effect of glutathione intake on exercise-induced muscle metabolism and fatigue in mice and humans.

Methods: ICR mice were divided into 4 groups: sedentary control, sedentary supplemented with glutathione (2.0\%, $5 \mathrm{\mu L} / \mathrm{g}$ body weight), exercise control, and exercise supplemented with glutathione. After 2 weeks, the exercise groups ran on a treadmill at $25 \mathrm{~m} / \mathrm{min}$ for $30 \mathrm{~min}$. Immediately post-exercise, intermuscular pH was measured, and hind limb muscle and blood samples were collected to measure biochemical parameters. In a double-blind, cross-over study, 8 healthy men $(35.9 \pm 2.0$ y) were administered either glutathione $(1 \mathrm{~g} / \mathrm{d})$ or placebo for 2 weeks. Then, they exercised on a cycle ergometer at 40\% maximal heart rate for $60 \mathrm{~min}$. Psychological state and blood biochemical parameters were examined after exercise.

Results: In the mouse experiment, post-exercise plasma non-esterified fatty acids were significantly lower in the exercise supplemented with glutathione group $(820 \pm 44 \mathrm{mEg} / \mathrm{L})$ compared with the exercise control group $(1152 \pm 61 \mathrm{mEq} / \mathrm{L})$. Intermuscular $\mathrm{pH}$ decreased with exercise $(7.17 \pm 0.01)$; however, this reduction was prevented by glutathione supplementation $(7.23 \pm 0.02)$. The peroxisome proliferator-activated receptor- $\gamma$ coactivator-1a protein and mitochondrial DNA levels were significantly higher in the sedentary supplemented with glutathione group compared with the sedentary control group ( $25 \%$ and $53 \%$ higher, respectively). In the human study, the elevation of blood lactate was suppressed by glutathione intake (placebo, $3.4 \pm 1.1 \mathrm{mM}$; glutathione, $2.9 \pm 0.6 \mathrm{mM}$ ). Fatigue-related psychological factors were significantly decreased in the glutathione trial compared with the placebo trial.
\end{abstract}

Conclusions: These results suggest that glutathione supplementation improved lipid metabolism and acidification in skeletal muscles during exercise, leading to less muscle fatigue.

Keywords: Glutathione, Dkeletal muscle, Lipid metabolism, Running exercise, PGC-1a

\footnotetext{
* Correspondence: waoi@kpu.ac.jp

'Laboratory of Health Science, Graduate School of Life and Environmental Sciences, Kyoto Prefectural University, 1-5 Hangi-cho Shimogamo, Sakyo-ku, Kyoto 606-8522, Japan

Full list of author information is available at the end of the article
} 


\section{Background}

Glutathione, a tripeptide consisting of glutamate, cysteine, and glycine, is synthesized primarily in the hepatic cells. It is stored in an oxidized or reduced form at high concentrations in the majority of cells. Glutathione is reported to be involved in the regulation of various physiological functions, in particular, antioxidation and detoxification functions [1,2]. Reduced glutathione is easily oxidized by reactive oxygen species and subsequently reduced again by glutathione reductase, and the redox balance of glutathione has been used as a marker of antioxidant status in various conditions. Physical exercise decreases the reduced form and increases the oxidized form of glutathione [3,4]. In addition, prolonged exercise decreases total plasma and tissue glutathione content over time [5,6], which suggests that glutathione may be associated with aerobic energy metabolism and maintenance of muscle contraction.

Energy and nutrient metabolism in skeletal muscle plays an important role in muscular fatigue. In particular, the energy source can affect muscle performance. For example, a carbohydrate-based energy source results in decreased muscular $\mathrm{pH}$ owing to increased lactic acid production, which leads to impaired muscle contraction. However, when the energy expended during exercise is derived from lipids, a large amount of energy can be continuously supplied via aerobic metabolism. Therefore, increased lipid utilization in the mitochondria of skeletal muscle cells is associated with continued muscle contraction, and the number and activity of mitochondria influence the utilization of fatty acids in muscle cells.

Over the past decade, peroxisome proliferator-activated receptor- $\gamma$ coactivator- $1 \alpha$ (PGC- $1 \alpha)$ has been demonstrated as a key transcriptional co-activator, providing a mechanistic insight into nuclear regulatory pathways in the biogenesis of mitochondria in skeletal muscle [7-9]. Its contents are changed by differing levels of physical activity, metabolic disorders, and aging, which are all associated with metabolic capacity [9-11]. Moreover, it interacts with nuclear receptors and transcription factors to activate the transcription of lipid metabolic genes, and its activity is responsive to aerobic metabolic events $[9,10]$.

Recently, Kovacs-Nolan et al. [12] demonstrated, in in vitro and ex vivo intestinal absorption model, that intact glutathione can be transported across human intestinal epithelial cells. In addition, we reported that the plasma glutathione concentration is transiently elevated after oral glutathione supplementation [13], which suggests that exogenous glutathione can be absorbed into the body and act as an important glutathione supply. Therefore, we hypothesized that glutathione supplementation may contribute to aerobic metabolism during exercise as a result of activated mitochondria via PGC$1 \alpha$ in skeletal muscle. The purpose of this study was to examine the effects of glutathione supplementation on muscle fatigue in exercise as a result of improved muscular aerobic metabolism.

\section{Methods}

\section{Animal experimental design}

This study complied with the guidelines of the Japanese Council on Animal Care and was approved by the Committee for Animal Research at Kyoto Prefectural University of Medicine (No. 23-77). ICR mice (7-week-old; Shimizu Laboratory Supplies, Kyoto, Japan) were acclimatized for 1 week in an air-conditioned $\left(22 \pm 2^{\circ} \mathrm{C}\right)$ room on a 12-h light/dark cycle (lights on from 7:30 to 19:30). Mice were divided into 4 groups containing 8 animals each: sedentary control, sedentary supplemented with glutathione, exercise control, and exercise supplemented with glutathione. Glutathione was purified and crystalized after separation from the fermented foodgrade yeast Candida utilis which approved by the U.S. food and drug administration (FDA), and used for experiments.

A glutathione solution $(2 \% \mathrm{w} / \mathrm{w})$ was provided 1 time per d ( $5 \mu \mathrm{L} / \mathrm{g}$ body weight) for 2 weeks. Saline was provided to control mice in the same volume. After 2 weeks of treatment, the exercise groups ran on a treadmill; after an initial 5-min warm up, the running speed was gradually increased to $25 \mathrm{~m} / \mathrm{min}$, which was maintained for $30 \mathrm{~min}$. Immediately following the exercise, intermuscular $\mathrm{pH}$ was measured under anesthesia; then the hind limb muscles and blood were collected. The control mice underwent the same measurements. Blood glucose was measured (GluTest; Arkray, Inc., Kyoto, Japan), following which the blood samples were centrifuged at $3,500 \mathrm{rpm}$ for $15 \mathrm{~min}$ at $4^{\circ} \mathrm{C}$ to collect plasma. Plasma nonesterified fatty acid (NEFA) was measured using a NEFA-C assay kit (WAKO, Osaka, Japan).

\section{$\mathrm{pH}$ measurements in animals}

$\mathrm{pH}$ levels in the interstitial fluid of exercised muscle tissue were measured under anesthesia using a glass microelectrode that was inserted into the interstitium between the gastrocnemius and tibialis anterior muscles.

\section{Western blotting}

Protein was extracted from muscle tissues obtained from sedentary groups using a lysis buffer (Sigma, St. Louise, $\mathrm{MO})$ containing protease inhibitor. Equal amounts of protein in the lysates were separated by $10 \%$ sodium dodecyl sulfate-polyacrylamide gel electrophoresis, and proteins were then transferred onto nitrocellulose membrane. The blots were incubated with primary antibodies against PGC-1 $\alpha$ (Chemicon International, Temecula, CA) and 
AMP-activated protein kinase (AMPK) (Cell Signaling Technology, Beverly, MA), and the reaction products were visualized using a horseradish peroxidase-conjugated secondary antibody (Invitrogen, Carlsbad, CA) and enhanced chemiluminescence (Chemi-Lumi One Super, Nakarai Tesque, Kyoto, Japan) (Additional file 1: Figure S1). Band densities were measured using ImageQuant LAS4000 (GE Healthcare, Buckinghamshire, UK).

\section{Mitochondrial DNA}

To determine the mitochondrial DNA (mtDNA), polymerase chain reaction (PCR) analysis was performed using DNA obtained from muscle tissues. Total DNA was extracted using the extraction reagent (DNA zol ${ }^{\circ} \mathrm{BD}$ Reagent, Invitrogen) following homogenization. The relative copy numbers of mitochondrial to nuclear DNA were determined by real-time PCR with primers specific to cytochrome c oxidase subunit II (COX II) (mitochondrial) 5' -ATCCCAGGCCGACTAAATCA (forward) and 5' -TT TCAGAGCATTGGCCATAGAA (reverse) and $\beta$-actin (nuclear) genes 5' -TATCCACCTTCCAGCAGATGT (forward) and 5' -AGCTCAGTAACAGTCCGCCTA (reverse).

\section{Human experimental design}

Eight healthy men (age, 35.9 \pm 2.0 y; height, $172.6 \pm$ $1.9 \mathrm{~cm}$; body weight, $70.6 \pm 3.2 \mathrm{~kg}$; BMI, $23.8 \pm 1.2 \mathrm{~kg} / \mathrm{m}^{2}$ ) were recruited to participate in a double-blind, cross-over study, which was complied with the principles outlined in the Helsinki Declaration and approved by the ethics committee of Kyoto Prefectural University (No. 46). All of the subjects provided written, informed consent. The subjects did not have a current or prior chronic disease or a history of smoking, and they were not currently using any medications. The subjects were also not habituated to a regular exercise regimen.

A single-bout exercise experiment was performed following 2 weeks of glutathione $(1 \mathrm{~g} / \mathrm{d})$ or placebo supplementation in capsule form. The subjects were asked to fast, except for water, from 22:00 the night prior to the experiment. On the experiment day, all subjects consumed the same breakfast (200 g of steamed rice) $90 \mathrm{~min}$ before the exercise session to normalize the effects of a pre-exercise meal. All participants performed a single bout of steady-state cycling exercise at $40 \%$ maximum heart rate for $60 \mathrm{~min}$. Heart rate and the rating of perceived exertion (RPE; Borg scale) were monitored every $10 \mathrm{~min}$ during exercise. Blood samples were collected from the antecubital vein before and after exercise, and blood glucose and lactate were measured using simple measuring instruments (Lactate Pro, GluTest; Arkray, Kyoto, Japan). Data obtained from seven subjects was analyzed for glucose and lactate due to failure of blood collection during exercise for one subject. Psychological state was assessed after blood collection using the
Profile of Mood State test, of which we were interested in 2 mood factors (fatigue-inertia and vigor-activity). In addition, the degree of relaxation was examined using a visual analogue scale (VAS). A 4-week washout period separated the conditions, and all of the subjects completed both testing conditions.

\section{Measurement of plasma glutathione}

Plasma glutathione concentration was measured according to our previously reported protocol [13]. Briefly, collected plasma was mixed with ethanol and then centrifuged. The supernatant was dried and added to $5 \%$ trichloroacetic acid (TCA) containing 2\% 2-mercaptoethanol to reduce the oxidized form of glutathione, and the mixture was used as the deprotenized plasma fraction. The ethanol precipitate of the plasma was mixed with the 5\% (final concentration) TCA and 2\% 2-mercaptoethanol. After centrifugation, the supernatant was used as the proteinbound plasma fraction. The concentrations of both fractions were determined by precolumn derivation with 6-aminoquinolyl-N-hydroxy succinimidyl carbamate and liquid chromatography-tandem mass spectrometry (LC-MS/MS).

\section{Statistical analysis}

All data are reported as mean \pm SE. Differences between groups or time course comparisons were evaluated using 2-way ANOVA. When significant interactions were detected, post hoc multiple comparisons were conducted using the Bonferroni method. Differences between the control and glutathione groups in the animal study were determined using Student's $t$-tests. Differences between the placebo and glutathione trials in the human study were tested using Wilcoxon signed-rank tests. $\mathrm{P}<0.05$ was considered statistically significant.

\section{Results}

Body weight and blood parameters in animals

Body weight was similar in all of the groups (sedentary control, $41.4 \pm 0.9 \mathrm{~g}$; sedentary supplemented with glutathione, $39.8 \pm 0.9 \mathrm{~g}$; exercise control $41.3 \pm 0.8 \mathrm{~g}$; exercise supplemented with glutathione, $40.2 \pm 0.7 \mathrm{~g}$ ). There were no significant differences in blood glucose levels between the control and glutathione groups in both sedentary and exercise conditions (degree of freedom $(\mathrm{df})=31, \mathrm{~F}=$ 0.039) (Figure 1A). In contrast, plasma NEFA after exercise was significantly lower with supplementation of glutathione compared with the control group $(\mathrm{df}=31, \mathrm{~F}=5.90$, $\mathrm{p}<0.01$ ) (Figure 1B).

\section{Intermuscular $\mathrm{pH}$ in animals}

The interstitial $\mathrm{pH}$ levels in muscle were significantly reduced by exercise $(\mathrm{df}=31, \mathrm{~F}=4.36, \mathrm{p}<0.001$; Figure $1 \mathrm{C})$. However, the $\mathrm{pH}$ following exercise of the glutathione 


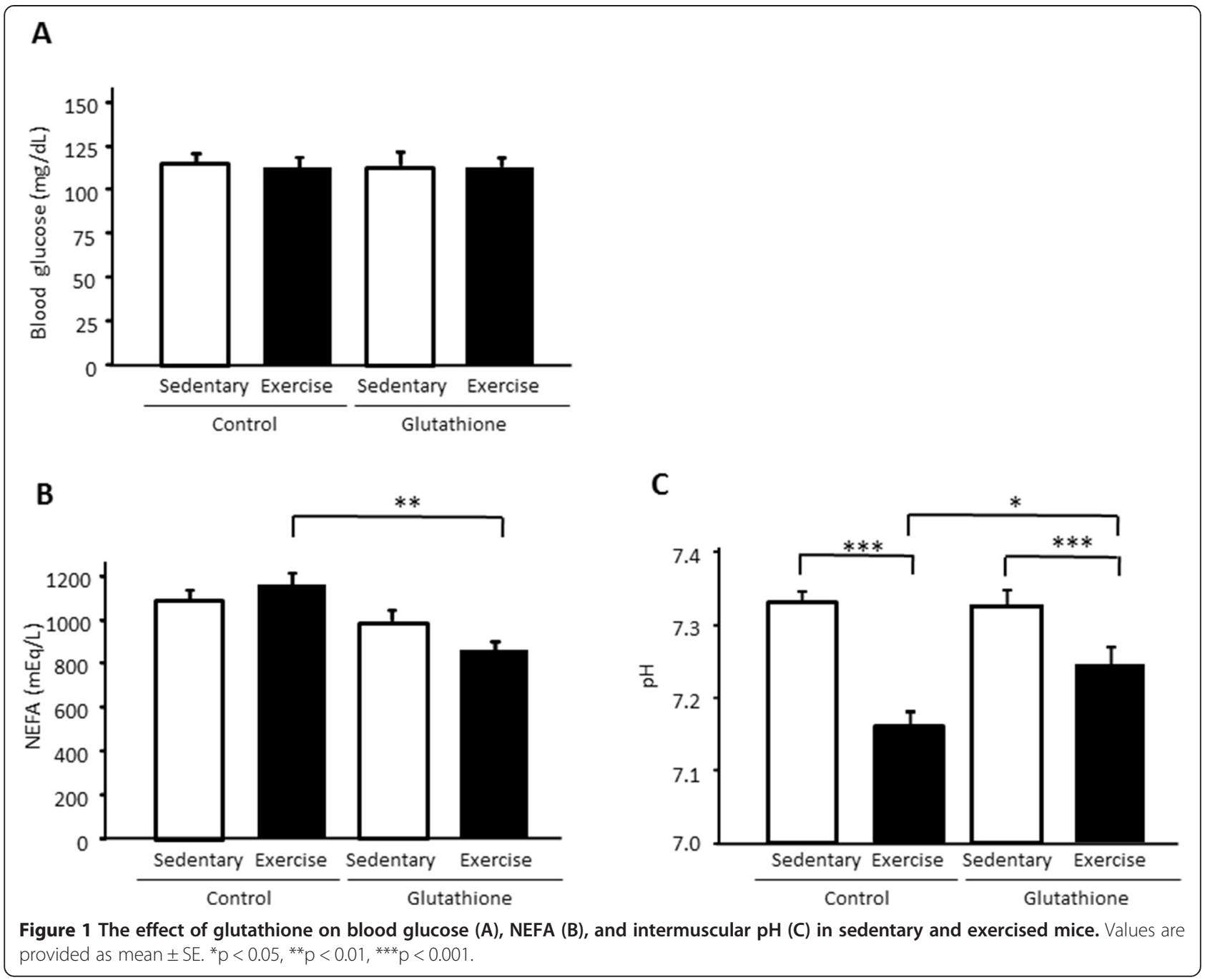

group was significantly higher than that of the control group $(\mathrm{p}<0.05)$.

PGC-1a, AMPK, and mitochondrial DNA in mouse muscle PGC-1 $\alpha$ was significantly higher with glutathione intake $(\mathrm{df}=14, \mathrm{t}=-1.88, \mathrm{p}<0.05 ;$ Figure $2 \mathrm{~A})$. In addition, AMPK, an upstream protein of PGC- $1 \alpha$, was also significantly higher in the sedentary treated with glutathione group than in the sedentary control group $(\mathrm{df}=13, \mathrm{t}=$ $-2.76, \mathrm{p}<0.05$; Figure $2 \mathrm{~B}$ ). In addition, mtDNA was significantly higher with glutathione supplementation $(\mathrm{df}=$ $14, \mathrm{t}=-1.98, \mathrm{p}<0.05$; Figure $2 \mathrm{C}$ ).

\section{Blood biochemical parameters in humans}

There was a significant decrease in the blood glucose concentrations at 30 and $60 \mathrm{~min}$ after exercise compared with pre-exercise in both the placebo and glutathione trials $(\mathrm{df}=41, \mathrm{~F}=23.9, \mathrm{p}<0.01)$ (Figure 3A); however, there was no difference between the trials. There was a significant increase in blood lactate concentrations at
30 min after exercise compared with pre-exercise in the placebo trial $(\mathrm{df}=41, \mathrm{~F}=3.90, \mathrm{p}<0.05)$ but not in the glutathione trial (Figure $3 \mathrm{~B}$ ). The free form of glutathione in plasma was not changed by either exercise or glutathione intake. In contrast, protein-bound plasma glutathione was significantly reduced by exercise in the placebo trial $(\mathrm{p}<0.05)$ although the reduction was moderated in the glutathione-supplemented group (Figure 4).

\section{Heart rate and psychological parameters in humans}

There was a trend for lower heart rates during exercise at 40 and $60 \mathrm{~min}$ in the glutathione trial compared with the placebo trial $(Z=-1.47, p=0.071$ and $Z=-1.26, p=$ 0.104 , respectively) (Figure 5 ). There was also a trend for a lower RPE at $50 \mathrm{~min}(\mathrm{Z}=-1.44, \mathrm{p}=0.075)$ and a significant decrease at $60 \min (Z=-1.78, p<0.05)$ in the glutathione trial compared with the placebo trial.

The Profile of Mood State vigor-activity factor after exercise was significantly higher following exercise in the glutathione trial compared with the placebo trial 

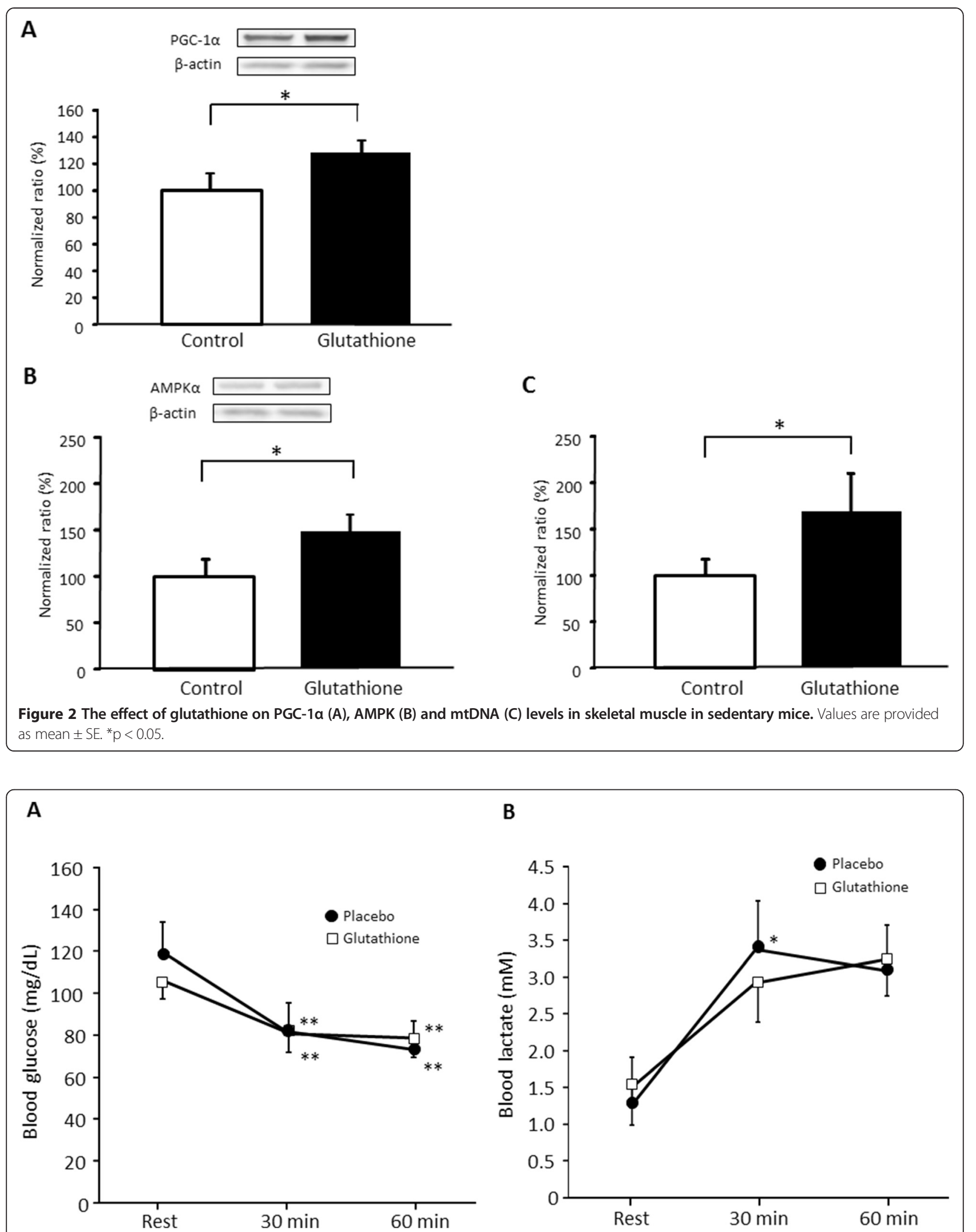

B

Figure 3 The effect of glutathione on blood glucose (A) and lactate (B) level in humans. Values are provided as the mean $\pm S E .{ }^{*} p<0.05$, ${ }^{* *} p<0.01$ vs. Rest. 


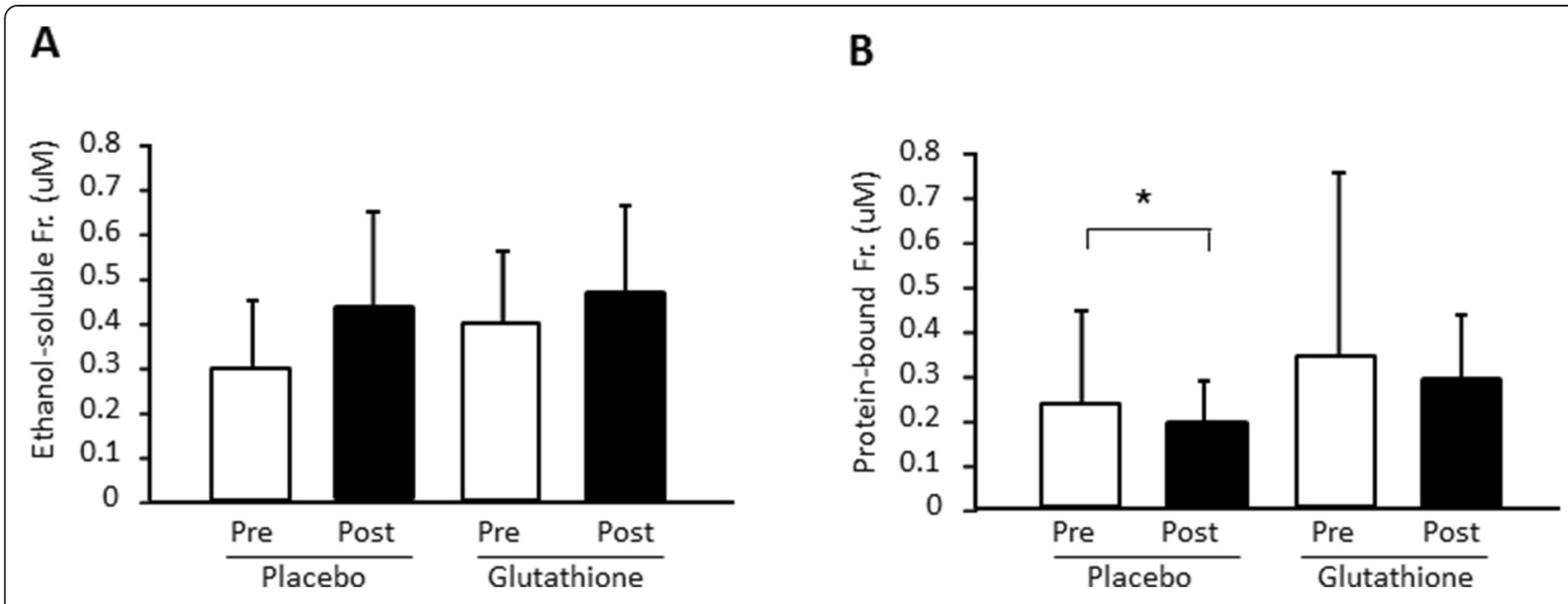

Figure 4 The effect of glutathione on plasma ethanol-soluble (A) and protein-bound (B) glutathione level in humans. Values are provided as the mean \pm SE. ${ }^{*} p<0.05$.

$(\mathrm{Z}=-2.11, \mathrm{p}<0.05)$ (Table 1$)$. In contrast, the fatigueinertia factor was significantly lower in the glutathione trial compared with the placebo trial $(\mathrm{Z}=-1.82, \mathrm{p}<$ $0.05)$, while marked difference was not found in the VAS scores between trials $(Z=-0.98, p=0.163)$.

\section{Discussion}

In the present study, glutathione supplementation resulted in higher levels of PGC-1 $\alpha$ and mtDNA biogenesis in mouse skeletal muscle and prevented the exercise-induced reduction in intermuscular $\mathrm{pH}$ in mice. Moreover, in humans, glutathione supplementation suppressed fatigue-related parameters during and after exercise. While it has been well documented that glutathione plays a central role in the antioxidant network in animal cells and redox balance can act as a marker of antioxidant status in various pathological and physiological conditions including exercise $[3,4,14,15]$, the role of exogenous glutathione on phenotypic changes relating to physical exercise has not been explored. To the best of our knowledge, the present study is the first to demonstrate that glutathione supplementation improves aerobic metabolism in skeletal muscle, leading to reduced exercise-induced muscle fatigue.

During muscle contraction, lactic acid, a major source of protons, is rapidly produced by increased glycolytic metabolism, lowering the $\mathrm{pH}$ and inhibiting muscle contraction [16]. The protons generated from cytosolic lactic acid are immediately buffered in the cell or exported to the interstitial fluid and further transported to the blood. The buffering capacity is relatively high in the cytosol and blood, whereas this capacity is low in interstitial fluid where the presence of buffering factors, such as proteins, is limited $[17,18]$. Therefore, the $\mathrm{pH}$ of the
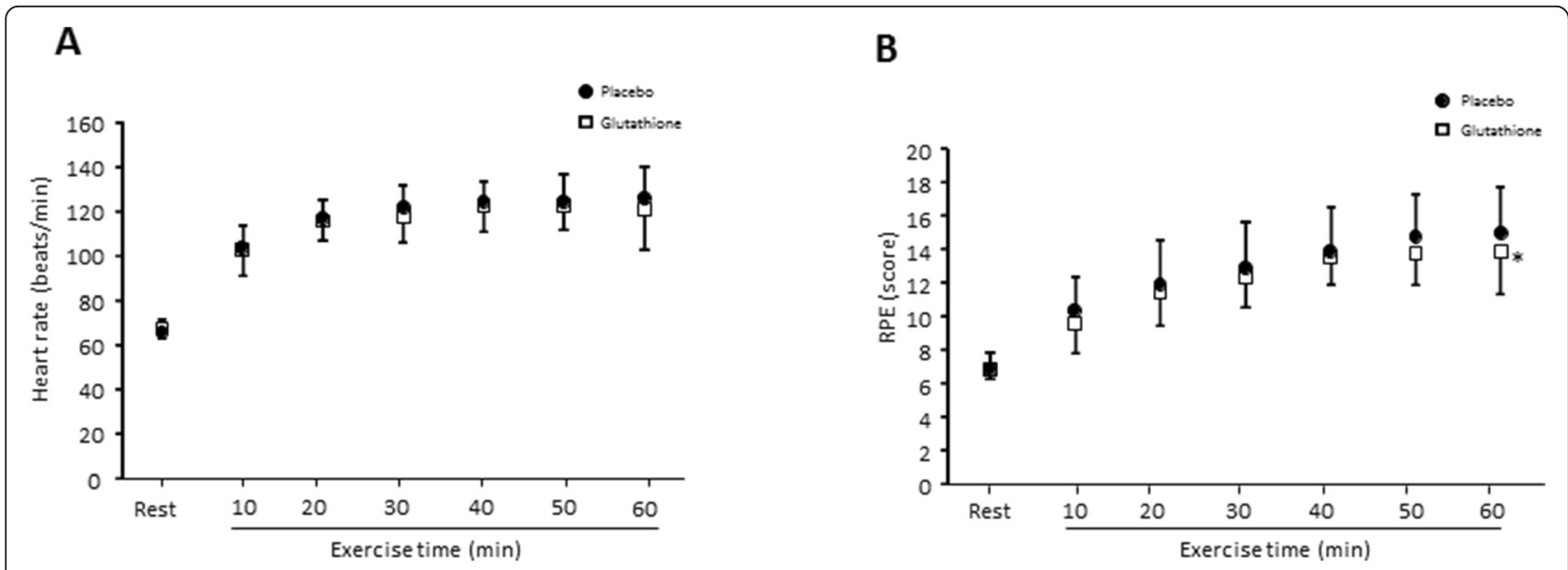

Figure $\mathbf{5}$ The effect of glutathione on heart rate (A) and the rating of perceived exertion (B) level in in humans. Values are provided as the mean \pm SE. * $p<0.05$ vs. placebo. 
Table 1 Psychological examination after exercise

\begin{tabular}{lll}
\hline & Placebo & Glutathione \\
\hline POMS test & & \\
$\quad$ Vigor-activity & $54.5 \pm 2.8$ & $58.8 \pm 3.2^{*}$ \\
Fatigue-inertia & $51.0 \pm 2.8$ & $45.1 \pm 2.7^{*}$ \\
VAS & $6.0 \pm 0.7$ & $4.7 \pm 0.8$ \\
\hline
\end{tabular}

Values are provided as mean $\pm \mathrm{SE}$. ${ }^{*} \mathrm{p}<0.05$ vs. placebo.

POMS, Profile of Mood State; VAS, visual analogue scale.

interstitial fluid in muscle tissues can drastically change in response to muscle contraction and can also be a marker of acid-base conditions in muscle tissue. In contrast, the majority of lactate anions are released into the circulation or immediately metabolized as an energy substrate through aerobic metabolism [19-21]; therefore, their levels are not suitable as a marker. The results of both the animal and human studies indicate that glutathione supplementation inhibited the decrease in intermuscular $\mathrm{pH}$ after exercise; in the human study, this was demonstrated by the differences in blood lactate concentrations following exercise between the placebo and glutathione trials. These results may also explain the differences observed between the trials in RPE and subjective fatigue during and after exercise; in particular, an improvement in muscular acidosis results in less fatigue.

Circulating NEFA concentrations are regulated by a balance between catabolic processes in adipose tissue and fatty acid substrate utilization by the skeletal muscle. Circulating catecholamines such as adrenalin and noradrenalin are increased in response to exercise and stimulate lipolysis of triglycerides in adipose tissue [22], which causes an elevation of circulating fatty acids. In contrast, muscle contraction increases uptake of fatty acids from the circulation into muscle cells [23], which leads to a decrease in circulating fatty acids. Therefore, we suggest that the reduction of NEFA observed in the glutathione-supplemented mice was due to an increase in muscle utilization rather than a release from adipose tissue. Because energy consumed in muscle during exercise is mainly supplied by carbohydrates and lipids, glutathione-induced lipid utilization can decrease energy obtained from carbohydrates, which may lead to a decrease in lactate/proton production. Collectively, this indicates that glutathione improves metabolic acidosis through the activation of lipid metabolism, which leads to suppression of exercise-induced fatigue.

PGC- $1 \alpha$ is a central member of a family of transcriptional co-activators involved in aerobic metabolism. Activation of PGC- $1 \alpha$ alters the metabolic phenotype through interactions with nuclear respiratory factor and peroxisome proliferator-activated receptor- $\alpha$ [8-10], which leads to increased mitochondrial biogenesis and activity. It has been reported that PGC-1 $\alpha$ activation causes significant improvements in athletic performance [24,25], prevention and treatment of muscle weakness in the elderly, obesity, and other metabolic diseases such as mitochondrial myopathies and diabetes $[10,11,26]$. Here, we detected an increase in PGC- $1 \alpha$ with 2 weeks of glutathione intake, along with an increase in mtDNA content, indicating the activation of mitochondrial biogenesis. Therefore, the observed elevation of PGC- $1 \alpha$ by glutathione intake strongly suggests an acceleration in lipid metabolism. In addition, the increase of mitochondria content could also lead to a decrease of lactate generation by accelerating aerobic metabolism of glucose, which would prevent muscle acidosis during exercise even further.

The regulatory mechanism for glutathione-induced increases in PGC-1 $\alpha$ is unclear. One explanation is the elevation of AMPK, which is an upstream factor of PGC- $1 \alpha$ regulation $[27,28]$. Recently, it has been argued that oral intake of other antioxidants, including vitamin $\mathrm{C}$ and $\mathrm{E}$, do not elevate PGC-1 $\alpha$ in the skeletal muscle of mice and humans [29,30]; thus, this may be a specific action of glutathione as a signal factor, but not its antioxidant properties. We found that 2 weeks of glutathione supplementation did not affect plasma glutathione concentration in the basal state. However, glutathione is transported across the intestines with in its intact form [12], and its plasma concentration, along with the glutathione-derived dipeptides $\gamma$-glutamyl-cysteine and cysteinyl-glycine, is markedly elevated during the $60-120$-min period after oral administration, as shown in our previous report [13]. Therefore, the transient elevation of glutathione or the derived dipeptides following supplementation over 2 weeks may indicate stimulation of specific signaling factors that lead to elevated AMPK and PGC- $1 \alpha$. Alternatively, glutathione content in muscle tissues may also increase with supplementation, leading to the up-regulation of these factors. Further studies are needed to determine the specific mechanism(s) by which glutathione affects muscle aerobic metabolism. In addition, we also observed that reduction of the protein-bound glutathione concentration in plasma after exercise was suppressed following glutathione supplementation, which may also be related to the regulation of energy metabolism or fatigue. Future studies should also aim to identify the bound protein in plasma and examine the mechanism of protein binding or release from the protein and its source.

\section{Conclusions}

The present results demonstrated that 2 weeks of glutathione supplementation decreased plasma fatty acids and suppressed the exercise-induced reduction in intermuscular $\mathrm{pH}$. Glutathione supplementation also resulted in elevated concentrations of PGC- $1 \alpha$ and mitochondria in skeletal muscle. These observations suggest that 
glutathione induces aerobic metabolism and improves an acidic environment in skeletal muscle during exercise by elevating PGC- $1 \alpha$, which would prevent exerciseinduced fatigue.

\section{Additional file}

Additional file 1: Figure S1. Images of western blotting for PGC-1a,

AMPK, and $\beta$-actin in control (C) and glutathione (G) groups.

\section{Abbreviations}

AMPK: AMP-activated protein kinase; COX II: Cytochrome c oxidase subunit II; mtDNA: Mitochondrial DNA; NEFA: Nonesterified fatty acid; PCR: Polymerase chain reaction; PGC-1 1a: Peroxisome proliferator-activated receptor- $\gamma$ coactivator-1a; RPE: Rating of perceived exertion; VAS: visual analogue scale.

\section{Competing interests}

This study was supported in part by a research fund from the KOHJIN Life Sciences Company, Ltd.

\section{Authors' contributions}

WA, YO, MT, YS, and AH conceived and designed the experiments. WA, YO, MT, EYP, SW, and KS performed research. TK, YS, EYP, and KS contributed reagents/materials/analysis tools. WA wrote the paper. All authors read and approved the final manuscript.

\section{Acknowledgements}

We would like to express our appreciation to the Kyoto Integrated Science \& Technology Bio-Analysis Center for the use of their LC-MS/MS.

\section{Author details}

'Laboratory of Health Science, Graduate School of Life and Environmental Sciences, Kyoto Prefectural University, 1-5 Hangi-cho Shimogamo, Sakyo-ku, Kyoto 606-8522, Japan. ${ }^{2} \mathrm{KOHJIN}$ Life Sciences Company, Ltd., Tokyo, Japan. ${ }^{3}$ Laboratory of Food Science, Graduate School of Life and Environmental Sciences, Kyoto Prefectural University, Kyoto, Japan. ${ }^{4}$ Division of Applied Biosciences, Graduate School of Agriculture, Kyoto University, Kyoto, Japan.

Received: 12 September 2014 Accepted: 6 January 2015

Published online: 06 February 2015

\section{References}

1. Meister A. Glutathione-ascorbic acid antioxidant system in animals. J Biol Chem. 1994;269:9397-400.

2. Tedeschi M, Bohm S, Di Re F, Oriana S, Spatti GB, Tognella S, et al. Glutathione and detoxification. Cancer Treat Rev. 1990;17:203-8.

3. Gambelunghe C, Rossi R, Micheletti A, Mariucci G, Rufini S. Physical exercise intensity can be related to plasma glutathione levels. J Physiol Biochem. 2001;57:9-14.

4. Ji LL, Fu R. Responses of glutathione system and antioxidant enzymes to exhaustive exercise and hydroperoxide. J Appl Physiol (1985). 1992;72:549-54.

5. Pyke $\mathrm{S}$, Lew $H$, Quintanilha A. Severe depletion in liver glutathione during physical exercise. Biochem Biophys Res Commun. 1986;139:926-31.

6. Lew H, Pyke S, Quintanilha A. Changes in the glutathione status of plasma, liver and muscle following exhaustive exercise in rats. FEBS Lett. 1985;185:262-6.

7. Wu Z, Puigserver P, Andersson U, Zhang C, Adelmant G, Mootha V, et al. Mechanisms controlling mitochondrial biogenesis and respiration through the thermogenic coactivator PGC-1. Cell. 1999;98:115-24.

8. Olesen J, Killerich K, Pilegaard H. PGC-1-alpha-mediated adaptations in skeletal muscle. Pflugers Arch. 2010;460:153-62.

9. Finck BN, Kelly DP. PGC-1 coactivators: inducible regulators of energy metabolism in health and disease. J Clin Invest. 2006;1 16:615-22.

10. Patti ME, Butte AJ, Crunkhorn S, Cusi K, Berria R, Kashyap S, et al. Coordinated reduction of genes of oxidative metabolism in humans with insulin resistance and diabetes: Potential role of PGC1 and NRF1. Proc Natl Acad Sci USA. 2003;100:8466-71.
11. Wenz T, Rossi SG, Rotundo RL, Spiegelman BM, Moraes CT. Increased muscle PGC-1alpha expression protects from sarcopenia and metabolic disease during aging. Proc Natl Acad Sci USA. 2009;106:20405-10.

12. Kovacs-Nolan J, Rupa P, Matsui T, Tanaka M, Konishi T, et al. In vitro and ex vivo uptake of GSH across the intestinal epithelium, and fate of oral GSH after in vivo supplementation. J Agric Food Chem. in press

13. Park EY, Shimura N, Konishi T, Sauchi Y, Wada S, Aoi W, et al. Increase in the protein-bound form of glutathione in human blood after oral administration of glutathione. J Agric Food Chem. 2014;62:6183-9.

14. Johnson WM, Wilson-Delfosse AL, Mieyal JJ. Dysregulation of glutathione homeostasis in neurodegenerative diseases. Nutrients. 2012;4:1399-13440,

15. Franco R, Schoneveld OJ, Pappa A, Panayiotidis MI. The central role of glutathione in the pathophysiology of human diseases. Arch Physiol Biochem. 2007;113:234-58.

16. Mainwood GW, Renaud JM. The effect of acid-base balance on fatigue of skeletal muscle. Can J Physiol Pharmacol. 1985;63:403-16.

17. Fogh-Andersen N, Altura BM, Altura BT, Siggaard-Andersen O. Composition of interstitial fluid. Clin Chem. 1995;41:1522-5.

18. Aukland K, Fadnes HO. Protein concentration of interstitial fluid collected from rat skin by a wick method. Acta Physiol Scand. 1973;88:350-8.

19. Brooks GA. The lactate shuttle during exercise and recovery. Med Sci Sports Exerc. 1986;18:360-8.

20. Bangsbo J, Aagaard T, Olsen M, Kiens B, Turcotte LP, Richter EA. Lactate and $\mathrm{H}+$ uptake in inactive muscles during intense exercise in man. J Physiol. 1995;488:219-29.

21. Juel C, Halestrap AP. Lactate transport in skeletal muscle - role and regulation of the monocarboxylate transporter. J Physiol. 1999;517:633-42.

22. Wahrenberg $\mathrm{H}$, Bolinder J, Arner P. Adrenergic regulation of lipolysis in human fat cells during exercise. Eur J Clin Invest. 1991;21:534-41.

23. Sahlin K, Harris RC. Control of lipid oxidation during exercise: role of energy state and mitochondrial factors. Acta Physiol (Oxf). 2008;194:283-91.

24. Calvo JA, Daniels TG, Wang X, Paul A, Lin J, Spiegelman BM, et al. Muscle-specific expression of PPAR-gamma coactivator-1alpha improves exercise performance and increases peak oxygen uptake. J Appl Physiol (1985). 2008;104:1304-12.

25. Tadaishi M, Miura S, Kai Y, Kano Y, Oishi Y, Ezaki O. Skeletal muscle-specific expression of PGC-1a-b, an exercise-responsive isoform, increases exercise capacity and peak oxygen uptake. PLoS One. 2011;6:e28290.

26. Zechner C, Lai L, Zechner JF, Geng T, Yan Z, Rumsey JW, et al. Total skeletal muscle PGC-1 deficiency uncouples mitochondrial derangements from fiber type determination and insulin sensitivity. Cell Metab. 2010;12:633-42.

27. Lee WJ, Kim M, Park HS, Kim HS, Jeon MJ, Oh KS, et al. AMPK activation increases fatty acid oxidation in skeletal muscle by activating PPARalpha and PGC-1. Biochem Biophys Res Commun. 2006;340:291-5.

28. Terada S, Goto M, Kato M, Kawanaka K, Shimokawa T, Tabata I. Effects of low-intensity prolonged exercise on PGC-1 mRNA expression in rat epitrochlearis muscle. Biochem Biophys Res Commun. 2002;296:350-4.

29. Ristow M, Zarse K, Oberbach A, Klöting N, Birringer M, Kiehntopf M, et al. Antioxidants prevent health-promoting effects of physical exercise in humans. Proc Natl Acad Sci USA. 2009;106:8665-70.

30. Gomez-Cabrera MC, Domenech E, Romagnoli M, Arduini A, Borras C, Pallardo FV, et al. Oral administration of vitamin $\mathrm{C}$ decreases muscle mitochondrial biogenesis and hampers training-induced adaptations in endurance performance. Am J Clin Nutr. 2008;87:142-9.

\section{Submit your next manuscript to BioMed Central and take full advantage of:}

- Convenient online submission

- Thorough peer review

- No space constraints or color figure charges

- Immediate publication on acceptance

- Inclusion in PubMed, CAS, Scopus and Google Scholar

- Research which is freely available for redistribution 